\title{
New Lower Bounds for the Randić Spread
}

\author{
Enide Andrade* \\ CIDMA-Center for Research and Development in Mathematics and Applications \\ Departamento de Matemática, Universidade de Aveiro, 3810-193, Aveiro, Portugal. \\ Maria Aguieiras A. de Freitas \\ Instituto de Matemática and COPPE/Produção \\ Universidade Federal do Rio de Janeiro, Rio de Janeiro, Brasil. \\ María Robbiano, Jonnathan Rodríguez \\ Departamento de Matemáticas, Universidad Católica del Norte Av. Angamos 0610 \\ Antofagasta, Chile.
}

\begin{abstract}
Let $G=(\mathcal{V}(G), \mathcal{E}(G))$ be an $(n, m)$-graph. The Randić spread of $G, s_{R}(G)$, is defined as the maximum distance of its Randić eigenvalues, disregarding the Randić spectral radius of $G$. In this work, we use numerical inequalities and bounds for the matricial spread to obtain relations between this spectral parameter and some structural and algebraic parameters of the underlying graph such as, the sequence of vertex degrees, the nullity, Randic index, generalized Randić indices and its independence number. In the last section a comparison is presented for regular graphs.
\end{abstract}

Keywords:

Matrix spread; Randić matrix, Randić spread, Normalized Laplacian spread, Laplacian spread, Regular graphs, nullity, independence number. 2000 MSC: 05C50, 15A 18

${ }^{*}$ Corresponding author

Email addresses: enide@ua.pt (Enide Andrade), maguieiras@im.ufrj.br (Maria Aguieiras A. de Freitas), mrobbiano@ucn.cl, jrodriguez01@ucn.cl (María Robbiano, Jonnathan Rodríguez) 


\section{Introduction}

This section is devoted to introduce some notation used throughout the text. We deal with an $(n, m)$-graph $G$ which is an undirected simple graph with vertex set $\mathcal{V}(G)$ of cardinality $n$ and edge set $\mathcal{E}(G)$ of cardinality $m$. If $e \in \mathcal{E}(G)$ has end vertices $i$ and $j$ we say that $i$ and $j$ are neighbors $(i \sim j)$ and we denote this edge by $i j$. The number of neighbors of vertex $i$ is denoted by $d_{i}$, and is called the degree of $i$. The minimum and maximum vertex degree of $G$ are denoted by $\delta(G)$ (or simply $\delta$ ) and $\Delta(G)$ (or simply $\Delta)$, respectively. A set of vertices that induces a subgraph with no edges is called an independent set and a maximum independent set of a graph $G$ is an independent set of largest cardinality $\alpha(G)$ which is called the independence number of $G$. A $k$-regular graph is a graph where every vertex has degree $k$. The complete graph of order $n$ is denoted by $K_{n}$. For a graph $G$ we denote by $G^{c}$ its complement. If $d_{i}=0$, for some $i$, then the corresponding vertex is an isolated vertex. Let $d_{1}, \ldots, d_{n}$ be the list of vertex degrees of $G$. The normalized Laplacian matrix of a graph $G$, denoted by $\mathcal{L}$, is a matrix whose rows and columns are indexed by the vertices of $G$, and its entries are described below:

$$
\mathcal{L}_{i j}= \begin{cases}1 & \text { if } i=j \text { and } d_{j} \neq 0 \\ -\frac{1}{\sqrt{d_{i} d_{j}}} & \text { if } i j \in \mathcal{E}(G) \\ 0 & \text { otherwise. }\end{cases}
$$

Similarly, the Randic matrix of a graph $G$, denoted by $R=R_{G}$, is a matrix whose rows and columns are indexed by the vertices of $G$ and having the following entries:

$$
R_{i j}= \begin{cases}\frac{1}{\sqrt{d_{i} d_{j}}} & \text { if } i j \in \mathcal{E}(G) \\ 0 & \text { otherwise. }\end{cases}
$$

The Randic matrix was used for the first time in 2005 by Rodríguez [32]. This matrix is closely related to the molecular structure-descriptor defined as

$$
\chi(G)=\sum_{i j \in \mathcal{E}(G)} \frac{1}{\sqrt{d_{i} d_{j}}},
$$

that was proposed by Milan Randic in 1975 and it usually referred as the Randić index, [17, 23, 24, 25, 31, 32]. To refer its importance, it is worth to say that this topological index is suitable for measuring the extent of 
the branching of the carbon-atom skeleton of saturated hydrocarbons. It is largely used in Chemistry but it also has applications in pharmacology and medicine namely, in the study of prediction of colon and breast cancer $([28])$. For a regular graph, $G$ with $n$ vertices it is easily checked that

$$
\chi(G)=\frac{n}{2} .
$$

In 1998 Bollobás and Erdös, [3], introduced the generalized randić index:

$$
R_{\alpha}(G)=\sum_{i j \in \mathcal{E}(G)}\left(d_{i} d_{j}\right)^{\alpha}
$$

for a real number $\alpha \neq 0$. Note that the Randic index defined in (1) is a particular case of the previous parameter considering $\alpha=-1 / 2$.

Most of the works related with bounds for $R_{\alpha}(G)$ focus on the case $|\alpha| \leq$ 1. In [26], the authors investigate bounds for $R_{\alpha}(G)$ considering $|\alpha|>1$. If $\delta \geq 1$, among other results, it was shown in [34] that

$$
\frac{n}{2 \Delta} \leq R_{-1}(G) \leq \frac{n}{2 \delta}
$$

where the equality holds if and only if $G$ is a regular graph.

Let us consider $A_{G}=\left(a_{i j}\right)$ the adjacency matrix of $G$. The spectrum of $A_{G}$ is called spectrum of $G$ and its elements are called the eigenvalues of $G$. Let $D_{G}$ (or simply $D$ ) denote the $n \times n$ diagonal matrix of the vertex degrees of $G$. For a graph $G$ without isolated vertices the Randić matrix (see e.g. $[4,5])$ can be written as

$$
R_{G}=D^{-1 / 2} A_{G} D^{-1 / 2}
$$

where $D^{-1 / 2}$ is the diagonal matrix whose $i$-th diagonal entry is the reciprocal of the square root of the degree $d_{i}$. It is also worth to recall that this matrix is symmetric and nonnegative. The Randic spectrum of $G$ is the spectrum of $R_{G}$. As a consequence of (4) for graphs without isolated vertices, the Randić matrix of $G$ has the same inertia as $A_{G}$, (see e.g. [20]). Furthermore, if e is the all ones vector and $\mathbf{w}=D^{1 / 2} \mathbf{e}$, where $D^{1 / 2}$ is the diagonal matrix whose $i$-th diagonal entry is the square root of $d_{i}$, then

$$
R_{G} \mathbf{w}=1 \mathbf{w}
$$

therefore $(1, \mathbf{w})$ is an eigenpair of $R_{G}$. 
The Laplacian matrix of $G, L_{G}=D_{G}-A_{G}$, is positive semidefinite and its spectrum is called the Laplacian spectrum of $G$. It is well known that $(0, \mathbf{e})$ is an eigenpair of $L_{G}$. For graphs without isolated vertices, the normalized Laplacian matrix is

$$
\mathcal{L}_{G}=I_{n}-R_{G} .
$$

For spectral properties related with this matrix, see e.g. [10]. Moreover, this matrix has connections with some interesting properties of graphs, see for instance [7] for its connections to Random walks.

If $G$ has no isolated vertices, $\mathcal{L}_{G}$ and $L_{G}$ are related as follows:

$$
\mathcal{L}_{G}=D^{-1 / 2} L_{G} D^{-1 / 2} .
$$

Therefore, the normalized Laplacian matrix has the same inertia as $L_{G}$, and thus it is a positive semidefinite matrix and from (5), the spectral radius of $R_{G}$ is the eigenvalue 1 . Moreover, $\mathbf{w}=D^{1 / 2} \mathbf{e}$ is an eigenvector of $\mathcal{L}_{G}$ associated with the eigenvalue 0 and its multiplicity becomes the number of connected components of $G$. Here, the eigenvalues of $R_{G}$ are ordered decreasingly $1=\rho_{1} \geq \rho_{2} \geq \cdots \geq \rho_{n}$ and the eigenvalues of $\mathcal{L}_{G}$ are ordered as $\gamma_{1} \geq \gamma_{2} \geq \cdots \geq \gamma_{n}=0$, where $\gamma_{i}=1-\rho_{n+1-i}$, with $1 \leq i \leq n$.

Let $\mu_{1} \geq \cdots \geq \mu_{n-1} \geq \mu_{n}=0$ be the Laplacian eigenvalues of $G$. In [12], it was defined the concept of Laplacian spread of $G$ as

$$
s_{L}(G)=\mu_{1}-\mu_{n-1} .
$$

Related with connected regular graphs the following lower bounds were obtained.

Theorem 1. [1, Theorem 4.2] Let $G$ be a connected $k$-regular graph with $n$ vertices. Then

$$
s_{L}(G) \geq \frac{2}{n} \sqrt{n+n k(n-k)-(k+1)^{2}} .
$$

Theorem 2. [8, Corollary 4.2] Let $G$ be a connected $k$-regular graph with $n \geq 2$ vertices. Then

$$
s_{L}(G) \geq \frac{2}{n-1} \sqrt{n k(n-k-1)},
$$

with equality if and only if $G$ is the complete graph $K_{n}$ or a conference graph. 
The normalized Laplacian spread of $G$ (see [7, Corollary 9] where it appears with no name and in $[15,18]$ for further references), is

$$
s_{\mathcal{L}}(G)=\gamma_{1}-\gamma_{n-1}
$$

In $[15,16]$, the concept of Randić spread of the graph $G, s_{R}(G)$, was defined as

$$
s_{R}(G)=\rho_{2}-\rho_{n},
$$

that is, the maximum absolute value of the difference between its pairwise distinct Randić eigenvalues, disregarding the Randić spectral radius. Some properties were studied, namely some lower and upper bounds for this spectral invariant were established. See e.g. [15, 16].

Remark 3. If $G$ has $\iota \geq 1$ isolated vertices then 0 is a normalized Laplacian eigenvalue with multiplicity at least $\iota$. In fact, previously referred, the multiplicity of the normalized Laplacian eigenvalue 0 of $G$ is related with the number of connected components of the graph. Thus, for a non-connected graph $G$ its normalized Laplacian spread coincides to its largest eigenvalue. On the other hand, when $G$ has at least one edge and isolated vertices the second largest Randic eigenvalue is greater than or equal to zero, and the least Randić eigenvalue is obtained from the Randić matrix constructed from a graph that is the union of non trivial connected components of $G$. In this case, the normalized Laplacian and Randić spread do not coincide. Consider for instance the graph $K_{3} \cup 5 K_{1}$. Its Randic eigenvalues are 0 with mutiplicity 5,1 with multiplicity 1 and $-\frac{1}{2}$ with multiplicity 2 . Then the Randić spread is $0+\frac{1}{2}$ and the Normalized Laplacian spread is $1-(-1 / 2)-0=3 / 2$. Therefore, from now on, and for simplicity, one only consider graphs without isolated vertices.

On the other hand, if $G$ is not connected then $s_{R}(G)=1-\rho_{n}=\gamma_{1}-0=$ $s_{\mathcal{L}}(G)$. From now on we only consider connected graphs. For the complete graph on $n$ vertices, its Randić eigenvalues are 1 and $-\frac{1}{n-1}$, where the negative one has multiplicity $n-1$. Thus, $s_{R}\left(K_{n}\right)=s_{\mathcal{L}}\left(K_{n}\right)=0$. In [15], it was shown that $K_{n}$ is the only connected graph $G$ such that $s_{R}(G)=0$.

In [15] it was proved that if $G$ is a $k$-regular graph, $k \geq 2$, then

$$
s_{L}(G)=k s_{R}(G) .
$$

The next theorem establishes a lower bound for $s_{R}(G)$ (and obviously for $\left.s_{\mathcal{L}}(G)\right)$ in function of $R_{-1}$. This lower bound was proved by S. Butler in [7]. 
Theorem 4. [7] Let $G$ be a connected graph. Then

$$
s_{R}(G)=s_{\mathcal{L}}(G) \geq \frac{2}{n-1} \sqrt{2(n-1) R_{-1}-n} .
$$

The next corollary is a direct consequence of Theorem 4 .

Corollary 5. Let $G$ be a connected graph with maximum vertex degree $\Delta$. Then

$$
s_{R}(G)=s_{\mathcal{L}}(G) \geq \frac{2}{n-1} \sqrt{\frac{n(n-1)}{\Delta}-n},
$$

with equality for $G=K_{n}$.

Moreover, as referred by S. Buttler in [7], if $G$ is $k$-regular $(k \geq 2)$ then, from previous theorem

$$
s_{R}(G)=s_{\mathcal{L}}(G) \geq \frac{2}{(n-1) k} \sqrt{n k(n-1-k)},
$$

(with equality for $G=K_{n}$ ), which reduces to the case given by F. Goldberg in [14] for regular graphs.

It is straightforward to check that, attending the relation in (8), the lower bound in (10) is derived from (7). Then we conclude that for the conference graph the lower bound (10) is also reached.

\section{Main Results}

In this section we present some new lower bounds for the Randić spread of a connected graph $G$, some of the results depending on the Randic index $\chi(G)$ or/and the generalized Randić index, $R_{-1}=R_{-1}(G)$.

Theorem 6. Let $G$ be a k-regular graph $(k \geq 2)$ with $n$ vertices. Then

$$
s_{R}(G) \geq \frac{2}{n k} \sqrt{n+n k(n-k)-(k+1)^{2}} .
$$

Equality holds for $G \cong K_{n}$.

Proof. By the equality in $(8), s_{R}(G)=\frac{s_{L}(G)}{k}$, now the result is a direct consequence of inequality in (6).

In [30] the following numerical inequality was presented. 
Theorem 7. [30] Let $a=\left(a_{1}, \ldots, a_{n}\right)$ and $b=\left(b_{1}, \ldots, b_{n}\right)$ two vectors with $0<m_{1} \leq a_{i} \leq M_{1}$ and $0<m_{2} \leq b_{i} \leq M_{2}, i=1, \ldots, n$, for some constants $m_{1}, m_{2}, M_{1}$ and $M_{2}$. Then,

$$
\frac{n^{2}}{4}\left(M_{1} M_{2}-m_{1} m_{2}\right)^{2} \geq\left(\sum_{i=1}^{n} a_{i}^{2}\right)\left(\sum_{i=1}^{n} b_{i}^{2}\right)-\left(\sum_{i=1}^{n} a_{i} b_{i}\right)^{2} .
$$

Remark 8. Note that, by Cauchy-Schwartz inequality the expression in the right hand side is always positive, for any suitable $a^{\prime} s$ and $b^{\prime} s$. This allows us to take the square root in the results below until Corollary 11.

Recall that, the nullity of a graph is the multiplicity of the zero eigenvalue of $G$. For some work related with this concept see for instance $[9,11,19]$. The next result presents a lower bound for $s_{R}(G)$ in function of $R_{-1}$ for a graph $G$ with $n$ vertices whose nullity is known and equals to $\mu$.

Theorem 9. Let $G$ be a connected graph with $n \geq 2$ vertices. Let $\mu$ be the nullity of $G$. Then

$$
s_{R}(G)=s_{\mathcal{L}}(G) \geq \frac{2 \sqrt{(n-\mu)\left(2 R_{-1}-1\right)-1}}{n-\mu} .
$$

The equality holds for the $n=p+q$ vertices complete bipartite graph $K_{p, q}$.

Proof. By hypothesis $A_{G}$ has $\mu$ null eigenvalues. In consequence, the matrix $R_{G}$ has $\mu$ eigenvalues equal to zero. In this case, and from the definition of Randić spread (that considers two by two distinct eigenvalues), the above set of eigenvalues can be reduced to the following set:

$$
\Xi=\{0\} \cup\left\{\rho \in \sigma_{R}(G): \rho \neq 1, \rho \neq 0\right\},
$$

with cardinality equals to $n-\mu$. At this point we use Theorem 7 replacing $a_{i}=1$ and $b_{i}=1-\rho_{i}$, for all $i$ such that $\rho_{i} \in \Xi$. Since $0<1 \leq a_{i} \leq 1$, and $0<1-\rho_{2} \leq b_{i} \leq 1-\rho_{n}$, for all $i$ such that $\rho_{i} \in \Xi$, we have $M_{1} M_{2}=1\left(1-\rho_{n}\right)$ and $m_{1} m_{2}=1\left(1-\rho_{2}\right)$. Therefore, by Theorem 7

$$
\sum_{i: \rho_{i} \in \Xi} 1 \sum_{i: \rho_{i} \in \Xi}\left(1-\rho_{i}\right)^{2}-\left(\sum_{i: \rho_{i} \in \Xi}\left(1-\rho_{i}\right)\right)^{2} \leq \frac{1}{4}(n-\mu)^{2}\left(\rho_{2}-\rho_{n}\right)^{2}
$$




$$
\begin{aligned}
& (n-\mu)\left(2 n+2 R_{-1}-2 \mu+1\right)-(n-\mu+1)^{2} \leq \frac{1}{4}(n-\mu)^{2}\left(\rho_{2}-\rho_{n}\right)^{2} \\
& \Longrightarrow \\
& 4(n-\mu)\left(2 R_{-1}-1\right)-1 \leq(n-\mu)^{2}\left(\rho_{2}-\rho_{n}\right)^{2} .
\end{aligned}
$$

Thus, the result follows.

Corollary 10. Let $G$ be a connected graph with $n \geq 2$ vertices. Let $\mu$ and $\Delta$ be the nullity of $G$ and the largest vertex degree, respectively. Then,

$$
s_{R}(G)=s_{\mathcal{L}}(G) \geq \frac{2 \sqrt{(n-\mu)\left(\frac{n}{\Delta}-1\right)-1}}{n-\mu} .
$$

Moreover, if $G$ is $k$-regular $(k \geq 2)$, then

$$
s_{R}(G)=s_{\mathcal{L}}(G) \geq \frac{2 \sqrt{(n-\mu)\left(\frac{n}{k}-1\right)-1}}{n-\mu} .
$$

The equality in formulas (13) and (14) is reached at $K_{n / 2, n / 2}$.

Proof. This result is a direct consequence of Theorem 9 and the left hand inequality in (3). In the case $G$ is $k$-regular we use

$$
R_{-1}(G)=\frac{n}{2 k}
$$

and $\Delta=k$, thus the result follows.

The next result presents a lower bound for $s_{R}(G)$ in function of $R_{-1}$ for a graph that has independence number $\alpha(G)$ not less than $\frac{n+1}{2}$.

Theorem 11. Let $G$ be a connected graph with $n \geq 2$ vertices with $\alpha(G) \geq$ $\frac{n+1}{2}$. Then,

$$
s_{R}(G)=s_{\mathcal{L}}(G) \geq \frac{\sqrt{2(n-\alpha(G))\left(2 R_{-1}-1\right)-1}}{n-\alpha(G)} .
$$

The equality holds for the $n$ vertices star $S_{n}$. 
Proof. Let $W=\left\{v_{1}, \ldots, v_{\ell}\right\}$ be an independent set of vertices with cardinality $\ell=\alpha(G)$. Let $H$ be a subgraph of $G$ induced by $W^{c}$. Then, its adjacency matrix takes the form

$$
A_{G}=\left(\begin{array}{cc}
0 & C \\
C^{T} & A_{H}
\end{array}\right) .
$$

Let $\alpha_{1} \geq \alpha_{2} \geq \cdots \geq \alpha_{n}$ and $\beta_{1} \geq \cdots \geq \beta_{\ell}$ be the eigenvalues of $A_{G}$ jointly with the eigenvalues of the matricial block $\mathbf{0}$, respectively. Then, by Cauchy interlacing Theorem [21], we have

$$
\alpha_{i} \geq \beta_{i} \geq \alpha_{n-\ell+i} \geq \beta_{n-\ell+i} \quad \forall i=1, \ldots, \ell .
$$

Thus,

$$
0=\alpha_{n-\ell+1}=\alpha_{n-\ell+2}=\cdots=\alpha_{\ell} .
$$

Therefore, $A_{G}$ has at least $2 \ell-n$ eigenvalues equal to zero and, in consequence the matrix $R_{G}$ has at least $2 \ell-n$ eigenvalues equal to zero. In this case, and from the definition of Randić spread (that considers two by two distinct eigenvalues), the above set of eigenvalues can be reduced to the following set:

$$
\Xi=\{0\} \cup\left\{\rho \in \sigma_{R}(G): \rho \neq 1, \rho \neq 0\right\} .
$$

Observe that, in this case we have $\mu \geq 2 \ell-n$ and therefore the cardinality of $\Xi$ is at most $2 n-2 \ell$. The proof follows in an analogous way as previous theorem.

Corollary 12. Let $G$ be a connected graph with $n \geq 2$ vertices with largest vertex degree $\Delta$. Suppose that $\alpha(G) \geq \frac{n+1}{2}$. Then,

$$
s_{R}(G)=s_{\mathcal{L}}(G) \geq \frac{\sqrt{2(n-\alpha(G))\left(\frac{n}{\Delta}-1\right)-1}}{n-\alpha(G)} .
$$

Moreover, if $G$ is $k$-regular $(k \geq 2)$, then

$$
s_{R}(G)=s_{\mathcal{L}}(G) \geq \frac{\sqrt{2(n-\alpha(G))\left(\frac{n}{k}-1\right)-1}}{n-\alpha(G)} .
$$


Proof. As $G$ is connected $\alpha(G) \leq n-1$. Moreover, $\Delta \leq n-1$ implies that $\frac{n}{\Delta} \geq 1$, then the expression under the square root is positive. This result is a direct consequence of Theorem 11 and the left hand inequality in (3). In the case $G$ is $k$-regular we use

$$
R_{-1}(G)=\frac{n}{2 k}
$$

and $\Delta=k$, thus the result follows.

In order to present another lower bound for the Randić spread (and in consequence for the normalized Laplacian spread) we recall a well known theorem due to Brauer, [6].

Theorem 13. [6] Let $M$ be an arbitrary $n \times n$ matrix with eigenvalues $\tau_{1}, \ldots, \tau_{n}$. Let $\mathbf{x}_{k}$ be an eigenvector of $M$ associated with the eigenvalue $\tau_{k}$, and let $\mathbf{q}$ be an arbitrary $n$-dimensional vector. Then the matrix $M+\mathbf{x}_{k} \mathbf{q}^{T}$ has eigenvalues $\tau_{1}, \ldots, \tau_{k-1}, \tau_{k}+\mathbf{x}_{k}^{T} \mathbf{q}, \tau_{k+1}, \ldots, \tau_{n}$.

Let $G$ be an $(n, m)$-graph with degree sequence $d_{1}, \ldots, d_{n}$. Since,

$$
\mathbf{w}=D^{1 / 2} \mathbf{e}=\left(\sqrt{d_{1}}, \ldots, \sqrt{d_{n}}\right)^{T},
$$

is an eigenvector of the normalized Laplacian matrix for the eigenvalue 0 , by Theorem 13, the matrix

$$
M(G)=\mathcal{L}_{G}+\beta \mathbf{w} \mathbf{w}^{T}
$$

has spectrum

$$
\begin{aligned}
\sigma(M(G)) & =\left\{\gamma_{1}, \ldots, \gamma_{n-1}\right\} \cup\left\{\beta \mathbf{w}^{T} \mathbf{w}\right\} \\
& =\left\{\gamma_{1}, \ldots, \gamma_{n-1}\right\} \cup\{2 m \beta\},
\end{aligned}
$$

where $\beta$ is an arbitrary real number. If $\gamma_{n-1} \leq 2 m \beta \leq \gamma_{1}$ then $s_{R}(G)=$ $s_{\mathcal{L}}(G)=s(M(G))$, where $s(M(G))$ stands for the matrix spread of $M$. See e.g. $[22,27,29]$.

Applying Theorem 7 to $M(G)$ in (19), the following result is obtained.

Theorem 14. Let $G$ be a connected $(n, m)$-graph with $n \geq 2$ vertices. Let $\gamma_{1} \geq \gamma_{2} \geq \cdots \geq \gamma_{n}=0$ be the normalized Laplacian eigenvalues of $G$. Let $\beta$ such that $\gamma_{n-1} \leq 2 m \beta \leq \gamma_{1}$. Then,

$$
s_{R}(G)=s_{\mathcal{L}}(G) \geq \frac{2}{n} \sqrt{2 n R_{-1}+4 \beta^{2}(n-1) m^{2}-4 n m \beta} .
$$


Proof. The proof uses Theorem 7 by replacing $a_{i}=1, b_{i}=\gamma_{i}$, for $1 \leq i \leq$ $n-1$. and $a_{n}=1, b_{n}=2 m \beta$. Since,

$$
\gamma_{n-1} \leq b_{i} \leq \gamma_{1}, \quad 1 \leq i \leq n
$$

we have, $m_{1}=1, m_{2}=\gamma_{n-1}, M_{1}=1$ and $M_{2}=\gamma_{1}$. Replacing in the inequality (11) one obtains

$$
\left(\gamma_{1}-\gamma_{n-1}\right)^{2} \geq \frac{4}{n^{2}}\left(n\left(\sum_{i=1}^{n-1} \gamma_{i}^{2}+4 \beta^{2} m^{2}\right)-\left(\sum_{i=1}^{n-1} \gamma_{i}+2 m \beta\right)^{2}\right)
$$

which implies

$$
\left(\gamma_{1}-\gamma_{n-1}\right)^{2} \geq \frac{4}{n^{2}}\left(n\left(n+2 R_{-1}+4 \beta^{2} m^{2}\right)-(n+2 m \beta)^{2}\right),
$$

and therefore

$$
\left(\gamma_{1}-\gamma_{n-1}\right)^{2} \geq \frac{4}{n^{2}}\left(2 n R_{-1}+4 \beta^{2}(n-1) m^{2}-4 n m \beta\right) .
$$

Let $g=2 n R_{-1}+4 \beta^{2}(n-1) m^{2}-4 n m \beta \Rightarrow g=\left(2 \beta m \sqrt{n-1}-\frac{n}{\sqrt{n-1}}\right)^{2}+$ $2 n R_{-1}-\frac{n^{2}}{n-1} \geq 0 \Leftrightarrow$

$$
\left(2 \beta m \sqrt{n-1}-\frac{n}{\sqrt{n-1}}\right)^{2} \geq \frac{n^{2}}{n-1}-2 n R_{-1} .
$$

Now we will proof that $\frac{n^{2}}{n-1}-2 n R_{-1} \leq 0$ which is true if and only if

$$
\frac{n^{2}}{n-1} \leq 2 n R_{-1} \Leftrightarrow \frac{n}{2(n-1)} \leq R_{-1}
$$

which always occurs because

$$
\frac{n}{2(n-1)} \leq \frac{n}{2 \Delta} \leq R_{-1}
$$

The above implies that the inequality (20) is true for all $\beta \in \mathbb{R}$. Hence, taking the square root at both sides, the result follows.

By considering the above result and the left hand inequality in (3) we derive the following corollary. 
Corollary 15. Let $G$ be a connected $(n, m)$-graph with $n \geq 2$ vertices and maximum vertex degree $\Delta$. Let $\gamma_{1} \geq \gamma_{2} \geq \cdots \geq \gamma_{n}=0$ be the normalized Laplacian eigenvalues of $G$. Let $\beta$ be such that $\gamma_{n-1} \leq 2 m \beta \leq \gamma_{1}$. Then

$$
s_{R}(G)=s_{\mathcal{L}}(G) \geq \frac{2}{n} \sqrt{\frac{n^{2}}{\Delta}+4 \beta^{2}(n-1) m^{2}-4 n m \beta} .
$$

Moreover, if $G$ is $k$-regular $(k \geq 2)$, then

$$
s_{R}(G)=s_{\mathcal{L}}(G) \geq 2 \sqrt{\frac{1}{k}+\beta^{2}(n-1) k^{2}-2 \beta k} .
$$

Proof. Note that, by analogous above argument we have

$s=\frac{n^{2}}{\Delta}+4 \beta^{2}(n-1) m^{2}-4 n m \beta=\left(2 \beta m \sqrt{n-1}-\frac{n}{\sqrt{n-1}}\right)^{2}+\frac{n^{2}}{\Delta}-\frac{n^{2}}{n-1} \geq 0$.

At this point the result follows from Theorem 14 and inequality (3)

Remark 16. Note that, in order to obtain new lower bounds for $s_{R}(G)=$ $s_{\mathcal{L}}(G)$, when $G$ is connected, different parameters $\beta$ such that $\gamma_{n-1} \leq 2 m \beta \leq$ $\gamma_{1}$, can be chosen. Moreover, since $s_{R}\left(K_{n}\right)=0$, unless one say the contrary, all the nontrivial (and here we mean greater than zero) lower bounds for $s_{R}(G)$ when $G$ is connected are obtained as equality cases for $G=K_{n}$.

Remark 17. The condition $\gamma_{n-1} \leq 2 m \beta \leq \gamma_{1}$ is equivalent to

$$
\frac{1-\rho_{2}}{2 m} \leq \beta \leq \frac{1-\rho_{n}}{2 m} \text {. }
$$

Thus

$$
\rho_{n} \leq 1-2 m \beta \leq \rho_{2} .
$$

Among other results, in [15] it was shown that

$$
\rho_{n} \leq-\frac{\chi(G)}{m} \leq \rho_{2}
$$

Thus $\beta=\frac{1}{2 m}\left(1+\frac{\chi(G)}{m}\right)$ satisfies the condition (21).

In [15] the following definition was given. 
Definition 18. [15] Let $G$ be an arbitrary $(n, m)$-graph with list of vertex degrees $d_{1}, \ldots, d_{n}$ such that $d_{i} \neq 0$ for all $1 \leq i \leq n$. For the $i$-th vertex of $G$, define

$$
\Gamma(i)=\sum_{j \sim i} \frac{1}{d_{j}}
$$

Clearly, if $G$ is a $k$-regular graph then $\Gamma(i)=1$, for all $1 \leq i \leq n$.

For an $n \times n$ normal matrix $M=\left(m_{i j}\right)$, in [2] it was introduced the following lower bound for the spread of $M$ that is an explicit function of the entries of the matrix,

$$
s(M)^{2} \geq \max _{i, j}\left\{\left(m_{i i}-m_{j j}\right)^{2}+2 \sum_{k \neq i}\left|m_{i k}\right|^{2}+2 \sum_{k \neq j}\left|m_{j k}\right|^{2}\right\} .
$$

If $m_{i i}=m_{j j}, \forall i \neq j$, it is clear that the maximum above is reached for $i=j=i_{0}$. In [15], applying this lower bound to the matrix

$$
B(G):=R_{G}-\beta \mathbf{w} \mathbf{w}^{T},
$$

where $\beta$ is as in (21) it was proved the following result.

Theorem 19. [15] Let $G$ be an arbitrary $(n, m)$-graph with list of vertex degrees $d_{1}, \ldots, d_{n}$, then

$$
s_{R}(G)^{2} \geq \max _{i, j}\left\{\Upsilon-4 \beta\left(d_{j}+d_{i}\right)(1-\beta m)-\beta^{2}\left(d_{j}+d_{i}\right)^{2}\right\},
$$

where $\Upsilon=\frac{2}{d_{j}} \Gamma(j)+\frac{2}{d_{i}} \Gamma(i)$ with $\Gamma(i)$ defined as in (23).

Taking into account that in inequality (24) the indices $i$ and $j$ can be equal, the following lower bound can be obtained.

Theorem 20. Let $G$ be an (n,m)-graph. Consider $\beta$ as in (21). Then

$$
s_{\mathcal{L}}(G)^{2}=s_{R}(G)^{2} \geq 4 \max _{i}\left\{\frac{1}{d_{i}} \Gamma(i)-2 \beta d_{i}(1-\beta m)-\beta^{2} d_{i}^{2} .\right.
$$


Proof. Taking $i=j=i_{0}$ in (26), the inequality (27) is quickly derived.

If $G \nsim K_{n}$ the parameter $\beta$ at Theorems 14 and 20 can be chosen as $\beta=\frac{1}{2 m}$ (see [16]). In the other cases, for any graph $G$ the parameter $\beta$ can be chosen as an entry of the following 3-tuple:

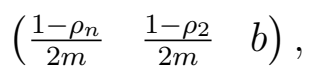

where $b=\frac{1}{2 m}\left(1+\frac{\chi(G)}{m}\right)$. Using these facts we obtain the following lower bounds for $s_{\mathcal{L}}(G)\left(=s_{R}(G)\right)$.

Corollary 21. Let $G \nsim K_{n}$ be a connected $(n, m)$-graph. Then

$$
s_{\mathcal{L}}(G)=s_{R}(G) \geq \frac{2}{n} \sqrt{2 n R_{-1}-n-1}
$$

Proof. Set $t=2 n R_{-1}-n-1$. Firstly we will proof that $t \geq 0$. Note that

$$
\begin{aligned}
t \geq 0 & \Longleftrightarrow 2 n R_{-1} \geq n+1 \\
& \Longleftrightarrow R_{-1} \geq \frac{n+1}{2 n}=\frac{1}{2}+\frac{1}{2 n},
\end{aligned}
$$

which is true since

$$
R_{-1} \geq \frac{n}{2 \Delta}
$$

and

as

$$
\frac{n}{2 \Delta} \geq \frac{1}{2}+\frac{1}{2 n}
$$

$$
\frac{n}{\Delta} \geq 1+\frac{1}{n} \Leftrightarrow \Delta \leq n-1
$$

At this point, the proof is obtained in a straightforward way replacing $\beta=\frac{1}{2 m}$ in the lower bound given at Theorem 14.

Corollary 22. Let $G \nsim K_{n}$ be a connected $(n, m)$-graph with maximum vertex degree $\Delta$. Then

$$
s_{\mathcal{L}}(G)=s_{R}(G) \geq \frac{2}{n} \sqrt{\frac{n^{2}}{\Delta}-n-1} .
$$

Moreover, if $G$ is k-regular

$$
s_{\mathcal{L}}(G)=s_{R}(G) \geq \frac{2}{n} \sqrt{\frac{n^{2}}{k}-n-1} .
$$


Proof. By the inequality

$$
\frac{n}{\Delta} \geq 1+\frac{1}{n}
$$

it follows that the quantity under the first above square root is positive. At this point the proof is obtained in a straightforward way by replacing $\beta=\frac{1}{2 m}$ in the lower bound given in Corollary 15.

Corollary 23. Let $G \nsim K_{n}$ be a connected $(n, m)$-graph with sequence of degrees $d_{1}, d_{2}, \ldots, d_{n}$. Then

$$
s_{\mathcal{L}}(G)^{2}=s_{R}(G)^{2} \geq 4 \max _{i}\left\{\frac{\Gamma(i)}{d_{i}}-\frac{d_{i}}{2 m}-\left(\frac{d_{i}}{2 m}\right)^{2}\right\} .
$$

Moreover, if $G$ is k-regular we re-obtain (28) as lower bound.

Proof. The proof is obtained in a straightforward way by replacing $\beta=\frac{1}{2 m}$ in the lower bound presented at Theorem 20 in (27). If $G$ is $k$-regular, from Definition 18 one can check that $\frac{\Gamma(i)}{d_{i}}=\frac{1}{k}, \forall i$.

Corollary 24. Let $G$ be a connected $(n, m)$-graph. Then

$$
s_{\mathcal{L}}(G)=s_{R}(G) \geq \frac{2}{n} \sqrt{2 n R_{-1}+\left(\frac{\chi(G)}{m}\right)^{2}(n-1)-\frac{2 \chi(G)}{m}-n-1} .
$$

Proof. The proof is obtained in a straightforward way by replacing $\beta=$ $\frac{1}{2 m}\left(\frac{\chi(G)}{m}+1\right)$ in the lower bound given in Theorem 14 .

Corollary 25. Let $G$ be a connected $(n, m)$-graph with maximum vertex degree $\Delta$. Then

$$
s_{\mathcal{L}}(G)=s_{R}(G) \geq \frac{2}{n} \sqrt{\frac{n^{2}}{\Delta}+\left(\frac{\chi(G)}{m}\right)^{2}(n-1)-\frac{2 \chi(G)}{m}-n-1} .
$$

Moreover, if $G$ is $k$-regular the lower bound for $s_{R}(G)$ coincides with the lower bound given in Theorem 6.

Proof. The proof is obtained in a straightforward way by replacing $\beta=$ $\frac{1}{2 m}\left(\frac{\chi(G)}{m}+1\right)$ in the lower bound given in Theorem 15. For a $k$-regular graph we replace $\Delta$ by $k$ and we take into account the equality in (2) and $m=\frac{n k}{2}$, thus the result holds. 
Corollary 26. Let $G$ be a connected $(n, m)$-graph with sequence of degrees $d_{1}, d_{2}, \ldots, d_{n}$. Then

$s_{\mathcal{L}}(G)^{2}=s_{R}(G)^{2} \geq 4 \max _{i}\left\{\frac{1}{d_{i}} \Gamma(i)-\frac{d_{i}}{2 m}\left(1-\left(\frac{\chi(G)}{m}\right)^{2}\right)-\left(\frac{d_{i}}{2 m}\right)^{2}\left(\frac{\chi(G)}{m}+1\right)^{2} \cdot\right\}$

Moreover, if $G$ is k-regular

$$
s_{\mathcal{L}}(G)=s_{R}(G) \geq 2 \sqrt{\frac{1}{k}-\frac{1}{n}\left(1-\frac{1}{k^{2}}\right)-\frac{1}{n^{2}}\left(1+\frac{1}{k}\right)^{2}} .
$$

Proof. The proof is obtained in a straightforward way by replacing $\beta=$ $\frac{1}{2 m}\left(\frac{\chi(G)}{m}+1\right)$ in the lower bound given in Theorem 20. The lower bound in (29) and the lower bound in Theorem 6 coincide.

\section{New Lower Bounds}

In this section, using some known numerical inequalities, lower bounds for the Randić spread of a graph with a prescribed degree sequence are presented. Some conclusions for a $k$-regular graph are obtained.

We start recalling the following numerical inequality, [33].

Lemma 27. [33] Let $a=\left(a_{1}, \ldots, a_{n}\right)$ and $b=\left(b_{1}, \ldots, b_{n}\right)$ be two positive $n$-vectors with $0<m_{1} \leq a_{i} \leq M_{1}$ and $0<m_{2} \leq b_{i} \leq M_{2}, i \in\{1, \ldots, n\}$, and constants $m_{1}, m_{2}, M_{1}, M_{2}$. The following inequality holds

$$
\frac{\sum_{i=1}^{n} a_{i}^{2}}{\sum_{i=1}^{n} a_{i} b_{i}}-\frac{\sum_{i=1}^{n} a_{i} b_{i}}{\sum_{i=1}^{n} b_{i}^{2}} \leq\left(\left(\frac{M_{1}}{m_{2}}\right)^{\frac{1}{2}}-\left(\frac{m_{1}}{M_{2}}\right)^{\frac{1}{2}}\right)^{2} .
$$

Remark 28. Note that, by the Cauchy-Swartz inequality the expression in the left hand side of the inequality (30) is nonnegative.

We introduce now more specific notation. For a square matrix $H, \operatorname{tr}(H)$ stands for the trace of $H$. The sum $\sum_{i_{1}=1}^{n} \sum_{i_{2}=1}^{n} \ldots \sum_{i_{s}=1}^{n} f\left(i_{1}, i_{2}, \ldots, i_{s}\right)$ is simply denoted by $\sum_{i_{1}, i_{2}, \ldots i_{s}=1}^{n} f\left(i_{1}, i_{2}, \ldots, i_{s}\right)$.

The next Lemma, jointly with the previous one, will be used to established a lower bound for the Randic spread of a graph with a prescribed degree sequence. 
Lemma 29. Let $\gamma_{1} \geq \gamma_{2} \geq \cdots \geq \gamma_{n-1} \geq \gamma_{n}=0$ be the normalized Laplacian eigenvalues of a graph $G$. Let $A=\left(a_{i j}\right)$ be the adjacency matrix of $G$ whose degrees sequence is $d_{1}, d_{2}, \ldots, d_{n}$. Then,

$$
\begin{aligned}
& \text { 1. } \sum_{i=1}^{n-1} \gamma_{i}^{2}=n+2 R_{-1} ; \\
& \text { 2. } \sum_{i=1}^{n-1} \gamma_{i}^{4}= \\
& \quad n+6 \sum_{i_{1}, i_{2}=1}^{n} \frac{a_{i_{1} i_{2}} a_{i_{2} i_{1}}}{d_{i_{1}} d_{i_{2}}}-4 \sum_{i_{1}, i_{2}, i_{3}=1}^{n} \frac{a_{i_{1} i_{2}} a_{i_{2} i_{3}} a_{i_{3} i_{1}}}{d_{i_{1}} d_{i_{2}} d_{i_{3}}}+\sum_{i_{1}, i_{2}, i_{3}, i_{4}=1}^{n} \frac{a_{i_{1} i_{2}} a_{i_{2} i_{3}} a_{i_{3} i_{4}} a_{i_{4} i_{1}}}{d_{i_{1}} d_{i_{2}} d_{i_{3}} d_{i_{4}}} .
\end{aligned}
$$

Proof. The equality in 1 . is obtained from the Frobenius matrix norm computation of $\mathcal{L}(G)$. For the second equality note that $\sum_{i=1}^{n-1} \gamma_{i}^{4}=\operatorname{tr}\left(\mathcal{L}(G)^{4}\right)$. Since

$$
\mathcal{L}(G)^{4}=I-4 R+6 R^{2}-4 R^{3}+R^{4},
$$

where $R=D^{-1 / 2} A D^{-1 / 2}$ is the Randić matrix of $G$, hence

$$
\operatorname{tr}\left(\mathcal{L}(G)^{4}\right)=\operatorname{tr}(I)-4 \operatorname{tr}(R)+6 \operatorname{tr}\left(R^{2}\right)-4 \operatorname{tr}\left(R^{3}\right)+\operatorname{tr}\left(R^{4}\right) .
$$

For $s \in \mathbb{N}$, the following equality is obtained.

$$
\operatorname{tr}\left(R^{s}\right)=\operatorname{tr}\left(\left(D^{-1} A\right)^{s}\right)=\sum_{i_{1}, i_{2}, \ldots i_{s}=1}^{n} \frac{a_{i_{1} i_{2}} a_{i_{2} i_{3}} \ldots a_{i_{s} i_{1}}}{d_{i_{1}} d_{i_{2}} \ldots d_{i_{s}}} .
$$

Therefore,

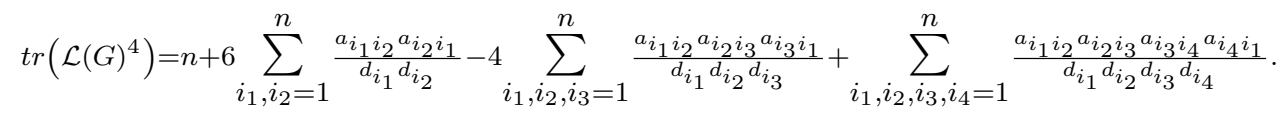

Thus, the equality at item 2 . holds.

As a consequence of the previous two lemmas we have the next result.

Theorem 30. Let $\gamma_{1} \geq \gamma_{2} \geq \cdots \geq \gamma_{n-1} \geq \gamma_{n}=0$ be the normalized Laplacian eigenvalues of a graph $G$. Let $A=\left(a_{i j}\right)$ be the adjacency matrix of $G$ whose degrees sequence is $d_{1}, d_{2}, \ldots, d_{n}$. Then

$$
s_{\mathcal{L}}(G)=s_{R}(G) \geq
$$




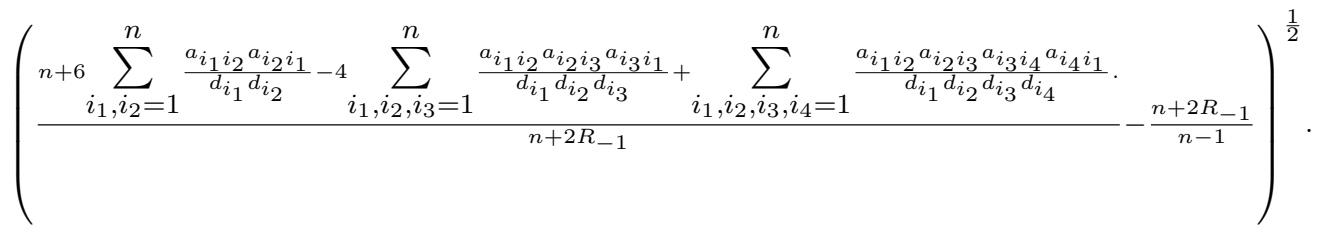

Proof. In this proof Lemma 27 is used replacing $a_{i}=\gamma_{i}^{2}$ and $b_{i}=1$, for $i=1, \ldots, n-1$. Note that,

$$
\gamma_{n-1}^{2} \leq \gamma_{i}^{2} \leq \gamma_{1}^{2} \quad(i=1, \ldots, n-1) .
$$

Moreover, $m_{1}=\gamma_{n-1}^{2}, M_{1}=\gamma_{1}^{2}$, and $m_{2}=1=M_{2}$. Replacing in the inequality (30) we obtain

$$
\frac{\sum_{i=1}^{n-1} \gamma_{i}^{4}}{\sum_{i=1}^{n-1} \gamma_{i}^{2}}-\frac{\sum_{i=1}^{n-1} \gamma_{i}^{2}}{n-1} \leq\left(\left(\gamma_{1}^{2}\right)^{\frac{1}{2}}-\left(\gamma_{n-1}^{2}\right)^{\frac{1}{2}}\right)^{2}
$$

Using identities 1. and 2. in Lemma 29 the result follows.

Applying Theorem 30, for a $k$-regular graph $G$ we obtain the following corollary.

Corollary 31. Let $\gamma_{1} \geq \gamma_{2} \geq \cdots \geq \gamma_{n-1} \geq \gamma_{n}=0$ be the Laplacian eigenvalues of a $k$ regular graph $G$. Let $A=\left(a_{i j}\right)$ be the adjacency matrix of $G$. Then

$$
\begin{gathered}
s_{\mathcal{L}}(G)=s_{R}(G) \geq \\
\left(\frac{n k+\left(1 / k^{3}\right) \operatorname{tr}\left(6 k^{2} A^{2}-4 k A^{3}+A^{4}\right)}{n(k+1)}-\frac{n(k+1)}{k(n-1)}\right)^{\frac{1}{2}} .
\end{gathered}
$$

Using (8) and (31) we obtain the following lower bound for the Laplacian spread.

Corollary 32. Let $G$ be a k-regular graph $(k \geq 2)$ with $n \geq 3$ vértices. Then

$$
s_{L}(G) \geq\left(\frac{n k^{3}+(1 / k) \operatorname{tr}\left(6 k^{2} A^{2}-4 k A^{3}+A^{4}\right)}{n(k+1)}-\frac{n k^{2}(k+1)}{k(n-1)}\right)^{\frac{1}{2}} .
$$


Finally, for a connected, $k$-regular graph $G$, we compare the lower bound given in Theorem 6 with its counterparts of the Corollary 22 .

Proposition 33. Let $G \nsim K_{n}$ be a connected k-regular graph. Then the lower bound given in Theorem 6 is an improvement of the lower bound given in Corollary 22 if and only if

$$
n \geq 2 k+1
$$

Proof. This proof is based on the equivalence

$$
\frac{2}{n k} \sqrt{n+n k(n-k)-(k+1)^{2}} \geq \frac{2}{n} \sqrt{\frac{n^{2}}{k}-n-1}=\frac{2}{n k} \sqrt{n^{2} k-n k^{2}-k^{2}},
$$

if and only if

$$
n \geq 2 k+1
$$

Acknowledgments. Enide Andrade was supported in part by the Portuguese Foundation for Science and Technology (FCT-Fundação para a Ciência e a Tecnologia), through CIDMA - Center for Research and Development in Mathematics and Applications, within project UID/MAT/04106/2013. M. Robbiano was partially supported by project VRIDT-UCN 16115 and she is grateful to hospitality of Universidade de Aveiro, Department of Mathematics, where this work was innitiated. J. Rodríguez was supported by CONICYT-PCHA/Doctorado Nacional/2015-21150477. M.A.A. de Freitas was partially supported by National Counsel of Technological and Scientific Development(CNPq-Conselho Nacional de Desenvolvimento Científico e Tecnológico) and FAPERJ (Fundação de Amparo à Pesquisa do Estado do Rio de Janeiro).

We want to thank the referee that pointed out a result in the first version of this work that already exists in the literature and was published by S. Butler in his Phd. Dissertation in 2008. Morever, we want to say that in the Chapter entitled An Overview on Randić (Normalized Laplacian Spread, of the book Bounds in Chemical Graph Theory - MATCH Commun. Math. Comput. Chem. the same result appears as Theorem 14 and Corollary 15 and should be referred as [7]. We also thank the referee for his/her valuable comments which led to an improvement of this paper. 
[1] E. Andrade, D. M. Cardoso, M. Robbiano, J. Rodriguez. Laplacian spread of graphs: lower bounds and relations with invariant parameters, Lin. Algebra Appl. 486 (2015): 494-503.

[2] E.R. Barnes, A.J. Hoffman. Bounds for the spectrum of normal matrices, Lin. Algebra Appl. 201 (1994): 79-90.

[3] B. Bollobás and P. Erdös. Graphs of extremal weights, Ars Combin. 50 (1998): 225-233.

[4] S. B. Bozkurt, A. D. Gungor, I Gutman. Randić matrix and Randić energy, MATCH Commun. Math. Comput. Chem. 64 (2010): 239-250.

[5] S. B. Bozkurt, A. D. Gungor, I Gutman. Randić spectral radius and Randić energy, MATCH Commun. Math. Comput. Chem. 64 (2010): 321- 334 .

[6] A. Brauer. Limits for the characteristic roots of a matrix. IV. Applications to stochastics matrices, Duke Math. J., 19-1 (1952): 75-91.

[7] S. Butler. Eigenvalues and structures of graph, Ph. Dissertation, Univ. California, San Diego, 2008.

[8] X. Chen, K. Ch. Das. Some results on the Laplacian spread of a graph, Lin. Algebra Appl. 505 (2016): 245-260.

[9] B.Cheng, B.Liu. On the nullity of graphs. Electron. Journal of Linear Algebra, vol. 16, article 5 (2007): 60-67.

[10] F. Chung. Spectral Graph Theory. CBMS Regional Conference Series in Mathematics 92, AMS, Providence, 1997.

[11] Dragoš M. Cvetkovič and Ivan M. Gutman. The algebraic multiplicity of the number zero in the spectrum of a bipartite graph. Matematicki Vesnik (Beograd), vol. 9, (1972): 141-150.

[12] Y. Z. Fan, J. Xu, Y. Wang, D. Liang, The Laplacian spread of a tree, Discr. Math. Theor. Comput. Sci. 10 (2008): 79-86.

[13] C, Godsil, G. Royle. Algebraic Graph Theory. Graduate texts in mathematics. Springer-Verlag 2001. 
[14] F. Goldberg. Bounding the gap between extremal Laplacian eigenvalues of graphs, Lin. Algebra Appl. 416 (2006): 68-74.

[15] H. Gomes, I. Gutman, E. A. Martins, M. Robbiano, B. San Martín. On Randic Spread, MATCH Commun. Math. Comput. Chem. 72 (2014): 249-266.

[16] H. Gomes, E. A. Martins, M. Robbiano, B. San Martín. Upper bounds on Randic spread, MATCH Commun. Math. Comput. Chem. 72 (2014): 267-278.

[17] I. Gutman, B. Furtula (Eds.), Recent Results in the Theory of Randić Index, Univ. Kragujevac, Kragujevac, 2008.

[18] I. Gutman, I. Milanović, E. Milanović, Bound for Laplacian-type graph energies. Miskolc Mathematical Notes. 16 (2015): 1 195-203.

[19] I. Gutman, B. Borovićanin, Nullity of graphs: an updated survey. Zbornik Radova (Beograd), vol. 14, 22 (Selected Topics on Applications of Graph Spectra), (2011): 137-154.

[20] E.V. Haynsworth. Determination of the inertia of a partitioned Hermitian matrix. Lin. Algebra Appl. 1 (1968): 73-81.

[21] W. Haemers. Interlacing eigenvalues and graphs. Lin. Algebra Appl (1995):593-616.

[22] E. Jiang, X. Zhan. Lower bounds for the Spread of a Hermitian Matrix, Lin. Algebra and Appl. 256 (1997): 153-163.

[23] X. Li, I. Gutman. Mathematical Aspects of Randić-Type Molecular Structure Descriptors, Univ. Kragujevac, Kragujevac, 2006.

[24] X. Li, Y. Shi. A survey on the Randić index, MATCH Commun. Math. Comput. Chem. 59 (2008): 127-156.

[25] X. Li, Y. Shi, L. Wang. An updated survey on the Randić index, in: I. Gutman, B. Furtula (Eds.). Recent Results in the Theory of Randić Index, Univ. Kragujevac, Kragujevac, (2008): 9-47.

[26] B. Liu, I. Gutman. On general Randić Indices, MATCH Commun. Math. Comput. Chem. 58 (2007): 147-154. 
[27] L. Mirsky. The spread of a matrix, Mathematika 3 (1956): 127-130.

[28] C.R. Munteanu, J.R. Rabual, J. Pereira. in Nuevas Fronteras Tecnolgicas. Redes Nanoroadmap e IBERO-NBIC 2010, Eds: J. Aguiló, A. Figueras, A. Freire, F. Martín, C. R. Munteanu, A. Pazos. Ciencia e Tecnologia para el Desarrollo (CYTED) Publisher, Madrid, Spain, ISBN 978-84-96023-82-6, Chapter: Prediccin del cncer colorretal com grafos (2010).

[29] P. Nylen, T. Y. Tam. On the spread of a hermitian matrix and a conjecture of Thompson, Lin. Multilin. Algebra 37 (1994): 3-11.

[30] N. Ozeki. On the estimation of the inequality by the maximum, J. College Arts, Chiba Univ. 5 (1968): 199-203.

[31] M. Randić. On characterization of molecular branching, J. Am. Chem. Soc. 97, (1975): 6609-6615.

[32] J. A. Rodríguez. A spectral approach to the Randić Index, Lin. Algebra Appl. 400 (2005): 339-344.

[33] O. Shiha, B. Mond. Bounds on differences of means, Inequalities I, NewYork- London (1967): 293-308.

[34] L. Shi. Bounds on Randić indices, Discr. Math. 309 (2009): 5238-5241. 